\title{
Adaptación de una Asignatura de Logopedia al Espacio Europeo de Educación Superior, EEES: Percepción de los Estudiantes
}

\author{
Blanca Laffon y Eduardo Pásaro \\ Universidad de A Coruña, Facultad de Ciencias de la Educación, Departamento de Psicología \\ (área Psicobiología), Campus Elviña s/n, 15071 A Coruña-España (e-mail: blaffon@udc.es)
}

Recibido Abr. 14, 2011; Aceptado May. 03, 2011; Versión final recibida May. 11, 2011

\begin{abstract}
Resumen
En este trabajo se describe la percepción de los estudiantes ante la adaptación de la asignatura Alteraciones Congénitas del Lenguaje perteneciente a la Diplomatura de Logopedia en la Universidad de La Coruña en España, al sistema europeo de transferencia de créditos. Los datos fueron obtenidos mediante encuestas cubiertas por los estudiantes del curso 2004-05 (créditos tradicionales) y 2007-08 (nuevo sistema de créditos). Los resultados muestran que ha mejorado su percepción respecto a las estrategias y recursos didácticos utilizados en las clases, valorando especialmente el enfoque práctico y el aprendizaje colaborativo. Sin embargo, también sugieren la necesidad de incrementar sus habilidades en ciertos aspectos necesarios para llevar a cabo un correcto aprovechamiento del trabajo realizado siguiendo las pautas del nuevo Espacio Europeo de Educación Superior, EEES.
\end{abstract}

Palabras clave: transferencia de créditos, EEES, enseñanza universitaria, recursos didácticos

\section{Adaptation of a Course from Speech Therapy Degree to the European Higher Education Area, EHEA: Students' Perception}

\begin{abstract}
The students' perception of the adaptation of the course Congenital Alterations of Language that belongs to the Speech Therapy Degree at the University of La Coruña in Spain, is studied and analyzed. The data were obtained by means of questionnaires filled in by students in 2004-05 (traditional credits) and in 2007-08 (new European system). The results showed that their perception has been enhanced with respect to the educational strategies and resources used in the classes, and that they especially value the practical focus and the collaborative learning. However, they also suggest the need of improving their abilities in certain aspects that are necessary to make a better use of the work performed in the new European Higher Education Area, EHEA.
\end{abstract}

Keywords: credit transfer, EHEA, higher education, educational resources 


\section{INTRODUCCIÓN}

Las bases para el establecimiento del Espacio Europeo de Educación Superior (EEES) fueron establecidas en la Declaración de Bolonia en 1999, fijándose como objetivo su implantación antes del año 2010. La implantación de los nuevos títulos comporta unos cambios significativos en la concepción de la formación académica, centrada en el aprendizaje más autónomo del alumno, adecuándose, en mayor medida, a las exigencias de la formación superior de la sociedad contemporánea. Así, en el EEES el aprendizaje se establece en base a competencias. El término competencia se define como el conjunto de conocimientos, actitudes y destrezas necesarias para desempeñar una ocupación dada (Monreal Gimeno, 2005). En el marco del EEES este término constituye un concepto complejo que engloba, por una parte, las características que definen la base de la personalidad del estudiante (competencias específicas), y que están asociadas a áreas de conocimiento concretas, y por otra parte las características relacionadas con la adquisición de conocimientos y habilidades (competencias genéricas), que pueden generarse en cualquier titulación (Martín y Bobb Wolff, 2011).

La consideración del aprendizaje a partir de competencias nos conduce a planificar nuestras asignaturas teniendo en cuenta el perfil profesional y las características particulares del alumnado. Además, significa preparar a nuestros estudiantes para que desarrollen un aprendizaje autónomo y que incorporen herramientas que vayan más allá de la mera adquisición de conocimientos, estimular el interés por saber más, enseñar a valorar de forma crítica la realidad y a tener un pensamiento reflexivo (Lázaro, 2004; García-Valcárcel, 2008). Esto implica una renovación que afecta a los procesos de enseñanza y aprendizaje, en los que están siendo fundamentales los equipos docentes en una doble vertiente: mejorar la calidad de la docencia impartida y ser un factor de cambio de la cultura laboral del profesorado y del personal al servicio de la docencia (Martínez y Viader, 2008).

Actualmente, la formación en competencias del alumnado permite la implantación de metodologías activas en el aula, lo que exige ensayar nuevas formas de enseñanza-aprendizaje y de sistemas de evaluación, que se adapten a las nuevas directrices europeas. En este sentido, coincidimos con Alfageme González (2007) cuando indica que los modos tradicionales de hacer en las aulas se deben modificar y, para ello, son necesarias grandes dosis de creatividad, espíritu crítico y la cultura del "razonar y reflexionar".

Uno de los primeros pasos a realizar en la adaptación al EEES y el diseño de asignaturas en base a formación en competencias fue la de adoptar el nuevo sistema europeo de transferencia de créditos (European Credit Transfer System, ECTS) en las titulaciones vigentes, como estadio previo a la implantación de los nuevos títulos de grado y postgrado. El sistema ECTS supone el uso de una de las herramientas más importantes para poder lograr todos los objetivos planteados para el EEES. El crédito ECTS representa en forma de valor numérico el volumen total de trabajo que el estudiante debe realizar para superar cada una de las asignaturas. Incluye clases teóricas, trabajos prácticos, seminarios, periodos de prácticas, trabajo de campo, trabajo personal (en bibliotecas o en el domicilio), así como los exámenes u otros posibles métodos de evaluación. Por ello, no se limita exclusivamente a las horas de asistencia o docencia presencial, constituyendo una medida del esfuerzo global realizado por el estudiante. Frente al crédito tradicional, que equivalía a 10 horas de clase presencial, el ECTS equivale a 25-30 horas de trabajo total del estudiante. En la Universidad de A Coruña (UDC) la equivalencia es de 25 horas.

En este trabajo se recogen los resultados obtenidos en la experiencia de adaptación de una asignatura de la Diplomatura de Logopedia de la UDC (Alteraciones Congénitas del Lenguaje) a los créditos ECTS durante el curso 2007-08, y su comparación con los créditos tradicionales, describiendo de forma detallada y desde diversos aspectos las percepciones del alumnado respecto a esta nueva metodología de trabajo, en donde ellos se convierten en actores principales del proceso de aprendizaje y adquisición de competencias. 


\section{METODOLOGÍA}

Los datos para la realización de este estudio se obtuvieron de las respuestas de los estudiantes matriculados en la asignatura "Alteraciones Congénitas del Lenguaje" de la Diplomatura en Logopedia de la UDC a preguntas recogidas en cuestionarios diseñados al efecto. Los cuestionarios fueron cubiertos por 25 estudiantes (80\% mujeres, $20 \%$ varones, edad media 21.1 \pm 1.6 años) a la finalización del curso académico 2004-05 (créditos tradicionales), y por 24 estudiantes (79\% mujeres, $21 \%$ varones, edad media $21.8 \pm 1.2$ años) a la finalización del curso académico 2007-08 (créditos ECTS). Se recogieron en ellos datos sobre el porcentaje de tiempo dedicado por los estudiantes a la realización de las diversas actividades comprendidas en el desarrollo de la asignatura, tanto antes de la implantación de los créditos ECTS como después. Además, en los cuestionarios cubiertos por los estudiantes del curso 2007-08 se incluyeron preguntas sobre diversos aspectos de la adaptación de la asignatura al EEES, como los recursos y estrategias didácticas empleados, sus propias habilidades para trabajar en el EEES y los aspectos en los que encuentran más dificultades o que más valoran.

Los datos obtenidos en los cuestionarios fueron analizados estadísticamente mediante la utilización del paquete informático SPSS (17.0). La normalidad de las variables estudiadas se determinó mediante el test de Kolmogorov-Smirnov. Dado que los datos se ajustaron a la distribución normal, se consideraron los tests paramétricos como adecuados para el análisis de los resultados. La existencia de diferencias entre los datos procedentes del curso 2004-05 y los procedentes del curso 2007-08 se investigó mediante el test $t$ de Student. El nivel de significación estadística se estableció en $P<0.05$.

\section{RESULTADOS Y DISCUSIÓN}

La metodología docente a utilizar en el marco del EEES debería aunar componentes didácticos que cumplan con los tres retos principales que se siguen de sus propósitos, es decir, deberían preparar a la nueva generación para sus papeles en el mundo del trabajo por adquisición de conocimiento y varias competencias, para participar como ciudadanos activos en una sociedad democrática y para poder asumir sus responsabilidades personales (Huber, 2008). En ese sentido, es preciso introducir innovación en la metodología docente que será utilizada en el marco del EEES. Así, coincidimos con Ruiz Ruiz (2008) en su apreciación de que la innovación debe formar parte de las actitudes vitales de los docentes, es decir, de los contenidos curriculares actitudinales que deben estar presentes en el currículo universitario. En base a esta filosofía, y antes de que la implantación de los nuevos Grados se hiciese efectiva, nos decidimos a llevar a cabo una experiencia de adaptación a los nuevos créditos ECTS de una asignatura de la Diplomatura de Logopedia de la UDC.

La asignatura Alteraciones Congénitas del Lenguaje figura en el plan de estudios de la Diplomatura de Logopedia de la Universidad de A Coruña con una carga docente de 4.5 créditos, de los cuales 3 son teóricos y 1.5 prácticos. Esto suponía, antes de la implantación de los créditos ECTS, que se establecía una duración del tiempo presencial de clases de teoría de 30 horas, y de clases prácticas de 15 horas. En el marco del EEES la Guía Docente tiene como objetivo principal orientar a los estudiantes en su proceso de aprendizaje, sobre todo en lo relativo a la búsqueda de la información y acerca del tiempo que deben utilizar para el desarrollo de las diferentes actividades programadas (Martín y Bobb Wolff, 2011). Por tanto, el primer paso en la adaptación fue preparar una Guía Docente, denominada en la UDC Guía GADU (Guía de Armonización de la Docencia Universitaria), en la que se recogiese un cambio en el planteamiento de las actividades académicas a realizar en la asignatura. Estas actividades se cuantifican en forma de créditos ECTS, que pretenden ser un reflejo de las horas totales de trabajo del estudiante, tanto presencial como no presencial. El resultado de este nuevo planteamiento se recoge en la Tabla 1, siguiendo el diseño de la Guía GADU. Las actividades académicas consisten fundamentalmente en clases magistrales, la preparación de trabajos en grupos por parte de los estudiantes, guiados por el profesor, que exponen oralmente ante la clase, la resolución de problemas en seminarios de debate, y la realización de un examen consistente en una prueba de respuesta múltiple, además de la atención personalizada en tutorías. 
Tabla 1: Planificación de las actividades académicas a realizar por el estudiante en la asignatura "Alteraciones Congénitas del Lenguaje" de la Diplomatura de Logopedia. En la Tabla, A es Horas presenciales y $B$ es la razòn (Horas no presenciales / trabajo autônomo)

\begin{tabular}{|c|c|c|c|c|c|c|}
\hline $\begin{array}{l}\text { Actividades } \\
\text { académicas }\end{array}$ & $\begin{array}{l}\text { Implica atención } \\
\text { personalizada }\end{array}$ & $\begin{array}{l}\text { Incluido en } \\
\text { evaluación }\end{array}$ & A & $\begin{array}{l}\text { Factor horas no } \\
\text { presenciales }\end{array}$ & B & $\begin{array}{c}\text { Horas totales } \\
\qquad A+B\end{array}$ \\
\hline $\begin{array}{l}\text { Presentación } \\
\text { oral }\end{array}$ & $\sqrt{\nabla}$ & $\sqrt{\nabla}$ & 6 & 1.5 & 9 & 15 \\
\hline \begin{tabular}{|ll||} 
Prueba & de \\
respuesta & \\
múltiple & \\
\end{tabular} & $\Gamma$ & $\sqrt{\checkmark}$ & 2 & 0 & 0 & 2 \\
\hline $\begin{array}{l}\text { Sesión } \\
\text { magistral }\end{array}$ & Г & Г & 20 & 2 & 40 & 60 \\
\hline \begin{tabular}{ll|} 
Solución & de \\
problemas & \\
\end{tabular} & Г & Г & 5 & 1.5 & 7.5 & 12.5 \\
\hline $\begin{array}{l}\text { Atención } \\
\text { personalizada } \\
\text { en tutorías }\end{array}$ & $\sqrt{\checkmark}$ & $\Gamma$ & 28 & 0 & 0 & 28 \\
\hline & & & & \multicolumn{3}{|c|}{ Horas totales: 112.5} \\
\hline & & & & Carga lectiva & éd & UDC: 4.5 \\
\hline
\end{tabular}

Según esta planificación, las 20 horas de clases magistrales no constituyen el mayor protagonismo de la actividad docente, que era lo que venía siendo habitual. Ahora es la atención personalizada al estudiante, realizada por parte del profesor en forma de tutorías, la que tiene ese mayor protagonismo. Además se recoge el tiempo invertido en la evaluación y presentación de trabajos monográficos en seminarios.

Los cuestionarios mediante los cuales se recogieron los datos necesarios para la realización de este trabajo fueron cubiertos por el $66 \%$ de los estudiantes en el caso de las encuestas realizadas en el curso 2004-05 (25 de 38 matriculados) y por el 72\% de los estudiantes en el caso del curso 2007-08 (24 de 33 matriculados). Los dos grupos de estudiantes resultaron similares en cuanto a distribuciones de edad y sexo, y también en cuanto a interés por la asignatura, ya que en el plan de estudios de la Diplomatura de Logopedia esta asignatura es optativa, mientras que en el Grado en Logopedia, que inició su implantación en la UDC en el curso 2009-10, la asignatura equivalente (Alteraciones de Base Congénita) es obligatoria. Durante el curso $2007-08$, el $74 \%$ de los estudiantes se sintieron protagonistas activos del proceso de aprendizaje, el $68 \%$ adquirieron conceptos que desconocían al trabajar en grupo y el $80 \%$ señalaron que habían dedicado más tiempo del que pensaban a la asignatura. En el curso 2004-05, para los mismos ítems los porcentajes habían sido del $42 \%, 26 \%$ y $65 \%$, respectivamente.

Los resultados obtenidos respecto a las actividades que implican presencia física del estudiante y profesor en el centro pueden observarse en la Figura 1A. Según ellos, la percepción que los estudiantes tienen sobre la porción de tiempo destinada para asistencia a sesiones magistrales desciende significativamente con la implantación de los nuevos créditos ECTS, así como aumenta significativamente el tiempo dedicado a las tutorías con el profesor. El papel del profesor en las tutorías consiste fundamentalmente en atender, facilitar y orientar al estudiante en su proceso formativo (De Miguel Díaz, 2005). De este modo, entendemos que la tutoría debe ser una actividad convenientemente programada en la adaptación a los créditos ECTS, con un contenido concreto y ofertada al estudiante como fórmula básica en su desarrollo académico. Por tanto, promovemos y facilitamos la participación del estudiante, siendo una actividad necesaria para la consecución de los objetivos planteados en la asignatura, y no pudiendo quedar solamente a merced de que el estudiante "sienta" la necesidad de asistir. Acorde con ello la participación en la tutoría se plantea a lo largo del período de formación de un modo sistemático y exigible, no esporádico o puntual. 
En la Figura 1B se recogen los resultados obtenidos respecto a las actividades académicas no presenciales. Como puede observarse, los estudiantes perciben que se reducen significativamente los tiempos dedicados a estudiar teoría y a la preparación de los exámenes, mientras que las actividades de búsqueda bibliográfica y consulta de la plataforma corporativa de teleformación de la UDC (Facultad Virtual) se incrementan de forma también significativa. A este respecto cabe mencionar que el uso adecuado de todas las posibilidades que esta herramienta telemática oferta, la actualización de sus contenidos, la atención individualizada a los estudiantes si se emplean foros de discusión o el correo electrónico como medio de comunicación profesorestudiante, suponen una dedicación de tiempo mayor y más continua que la que se emplea habitualmente en la preparación de clases teóricas o prácticas (Moreno y Santiago, 2003).

Por otra parte, a pesar de que podría inicialmente pensarse que la utilización de la teleformación puede constituir un obstáculo importante para estudiantes poco familiarizados o menos predispuestos hacia el uso de estas tecnologías, nuestros resultados contradicen claramente este supuesto. Por tanto, no cabe duda de que las nuevas tecnologías están transformando progresivamente el ambiente del aula y las funciones docentes, y estos cambios están induciendo una transformación sistemática en las teorías y prácticas didácticas.
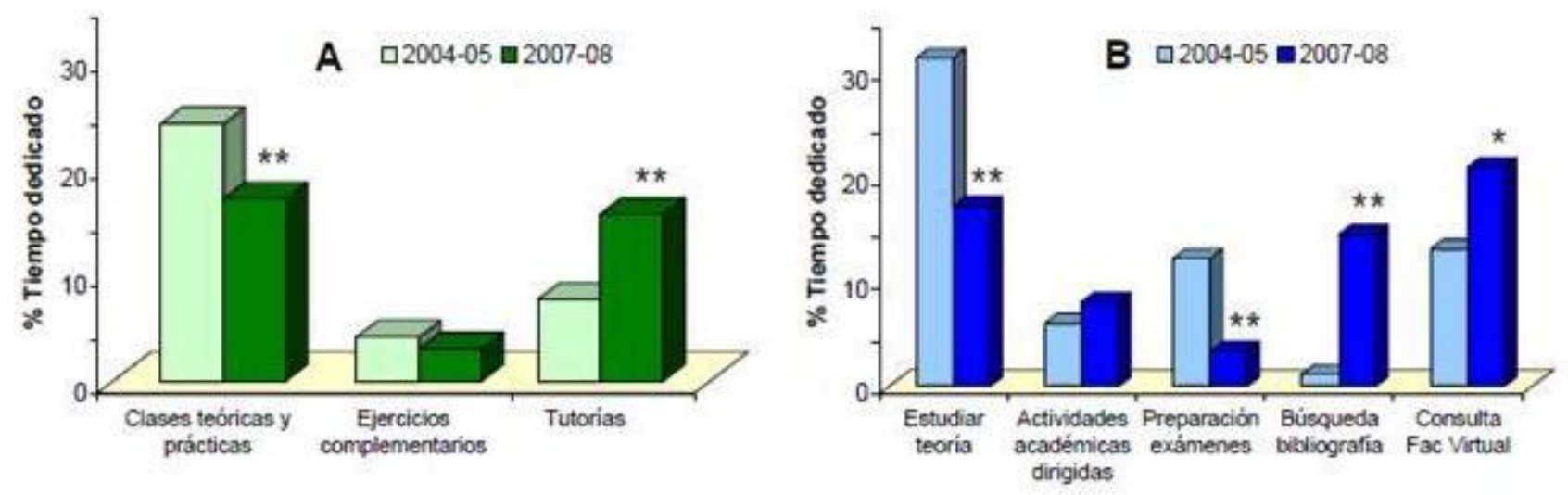

Fig. 1: Tiempo dedicado por los estudiantes a la realización de actividades académicas presenciales (A) y no presenciales (B) en la asignatura "Alteraciones Congénitas del Lenguaje". ${ }^{* \star} P<0.01$, diferencias significativas respecto al curso 2004-05.

A continuación se describen los resultados de las preguntas incluidas únicamente en los cuestionarios de los estudiantes del curso 2007-08, que versaban sobre diversos aspectos del trabajo en el EEES. Los resultados de las preguntas centradas en la comparación de las estrategias y recursos didácticos utilizados tras la adaptación al EEES con los empleados tradicionalmente se recogen en la Figura 2A. En cuanto a las tutorías, la realización de trabajos monográficos y el sistema de evaluación utilizado, los estudiantes percibieron una clara mejoría tras la adaptación al EEES, siendo bastante similares los porcentajes de individuos que no apreciaron diferencias y los que estimaron que han experimentado empeoramiento. Así, detectamos que en general los estudiantes valoran positivamente el mayor tiempo dedicado a las tutorías y los nuevos instrumentos utilizados en la generación de evidencias evaluativas para fomentar la adquisición de competencias (Villardón, 2006). En lo que respecta a las clases magistrales, la opinión mayoritaria (62\%) se decantó por la no existencia de variaciones tras la adaptación, mientras que en cuanto a la utilidad de la plataforma virtual en el EEES, la mayoría de las estimaciones estuvo igualmente repartida entre la mejoría y la igualdad ( $37 \%$ ambas).

En general, los aspectos que más valoraron los estudiantes de la adaptación al EEES (Figura 2B) fueron la posibilidad de trabajar sobre problemas cotidianos y buscar soluciones conjuntas, la aportación de puntos de vista diferentes sobre cuestiones del programa, la orientación participativa del curso y su enfoque esencialmente práctico. Palomares Ruiz (2011) realizó recientemente un estudio en la Universidad de Castilla la Mancha que comparte algunas características con el nuestro. Describe el uso de nuevas metodologías en la enseñanza, aprendizaje y evaluación, utilizando asimismo ECTS para representar el volumen efectivo de 
trabajo del alumnado. Según sus resultados, el grado de satisfacción del trabajo realizado por los estudiantes es muy alto (85\%), valorando la experiencia globalmente como excelente o muy gratificante el $87 \%$ de ellos, aunque un gran porcentaje $(74 \%)$ subraya que requiere una mayor dedicación que las metodologías tradicionales.
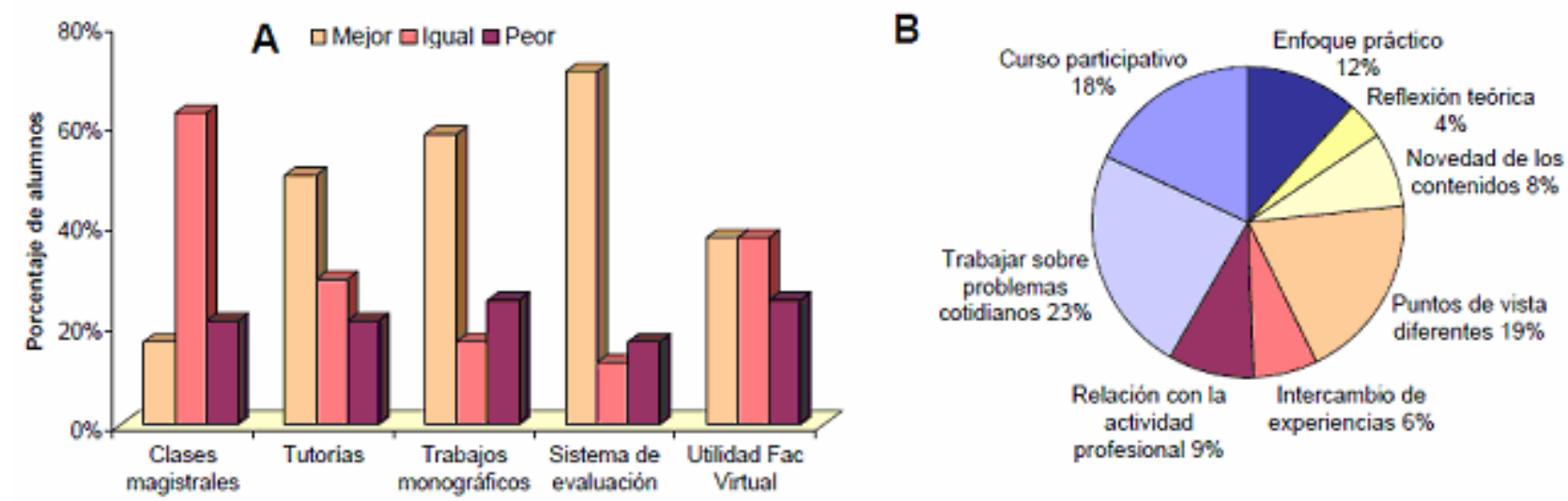

Fig. 2: Consideraciones de los estudiantes del curso 2007-08 sobre las estrategias y recursos didácticos adaptados al EEES respecto a los utilizados tradicionalmente (A) y los aspectos del curso más valorados (B).

De entre los factores que los estudiantes de nuestro estudio estimaron que les dificultan la realización de las tareas derivadas del EEES, la dificultad para organizar todos los trabajos, la falta de motivación y el elevado esfuerzo personal estuvieron igualmente representados (aproximadamente un 30\%) (Figura 3A). En cuanto a la percepción de los estudiantes sobre las habilidades que consideran que deben mejorar para trabajar en el EEES (Figura 3B), la mejora del trabajo en grupo y la coordinación con otros compañeros fueron las mayoritariamente representadas, seguidas muy de cerca por el manejo de TIC (herramientas de trabajo del tipo de bases de datos, plataformas tecnológicas colaborativas, etc.) y la planificación del trabajo no presencial. Actualmente existe un acuerdo unánime sobre la importancia de fomentar las competencias y habilidades de trabajo en grupo en la Universidad, ya que en el desarrollo de la vida profesional se trabaja a menudo en equipo para lograr objetivos comunes (García-Montalvo, 2001).

Ruiz Ruiz (2008) describe una experiencia de diseño de una asignatura de Pedagogía de la Universidad Complutense de Madrid por competencias en el marco del EEES. En su estudio, en el que se potencia el trabajo en equipo y la capacidad para motivar y crear ilusión en los alumnos como las claves del éxito de un aprendizaje significativo, los estudiantes valoraron muy positivo el grado de viabilidad y desarrollo de las competencias trabajadas. Sin embargo, y coincidiendo con nuestros resultados, reconocen que las competencias como el trabajo autónomo, la gestión del tiempo, la creación de nuevas ideas y trabajo en equipo necesitan ser reforzados para obtener el nivel óptimo. Asimismo, en el estudio realizado por Palomares Ruiz (2011) se comprobó que el alumnado realiza numerosas actividades autónomas relacionadas con la asignatura, dedicando mucho tiempo a ello; sin embargo, no las comparte con otros compañeros. En este sentido, la autora sugiere que se precisa crear el contexto adecuado para intercambiar las experiencias y aprendizajes producidos fuera del aula mediante aprendizaje autónomo.

Por último, las opiniones de nuestros estudiantes estuvieron muy divididas en cuanto a la estimación de si su formación sería mejor si todas las asignaturas de la titulación estuvieran adaptadas al EEES, ya que el $37 \%$ estimó que sí y el $54 \%$ opinó que no. Sin embargo, unánimemente $(79 \%)$ se decantaron por que el trabajo que tienen que realizar es superior con el nuevo EEES. Además, valoraron de forma global la calidad de la formación recibida calificándola como excelente (8\%), buena (59\%), regular (29\%) o mala (4\%). 
A

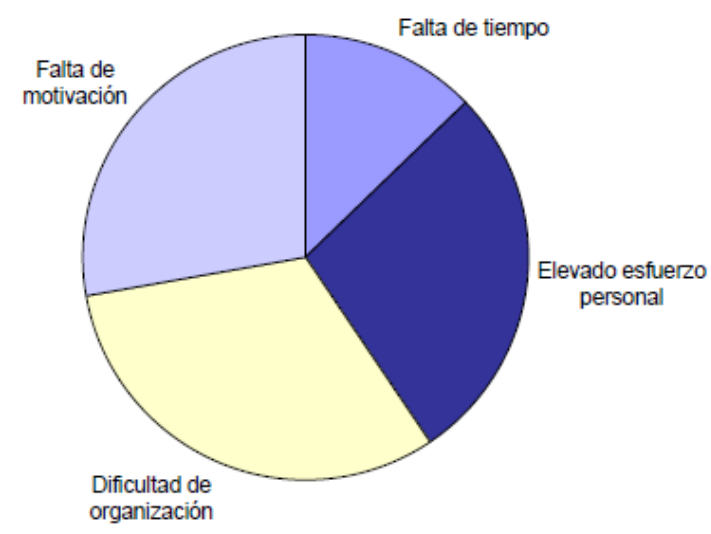

B

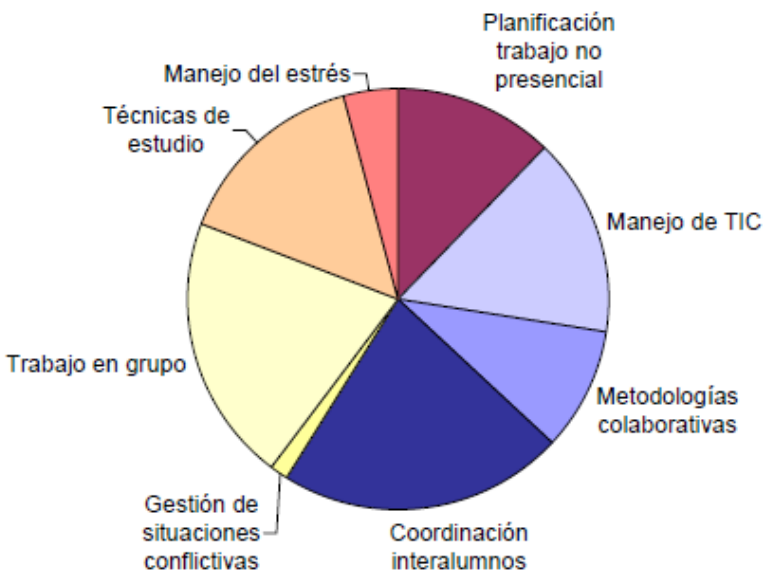

Fig. 3: A: Percepción de los estudiantes sobre los factores que les dificultan la realización de tareas en el EEES (A); B: aspectos en los que deben adquirir más habilidades para trabajar en el EEES

\section{CONCLUSIONES}

La adaptación de la asignatura "Alteraciones Congénitas del Lenguaje" al EEES ha supuesto un cambio en la metodología docente y en la forma de trabajo de los estudiantes, ganando protagonismo las tutorías, la consulta de fuentes bibliográficas y la utilización de medios de teleformación, en detrimento del tiempo dedicado al estudio y preparación de exámenes. En general ha mejorado su opinión respecto a las estrategias y recursos didácticos utilizados en las clases, valorando especialmente el enfoque práctico y el aprendizaje colaborativo. Por tanto, los resultados obtenidos muestran en general la mayor conformidad de los estudiantes con esta nueva forma de trabajo, aunque también sugieren la necesidad de incrementar sus habilidades en ciertos aspectos necesarios para llevar a cabo un correcto aprovechamiento del trabajo realizado en el EEES, especialmente en lo referido a la coordinación interalumnos, la realización de trabajos en grupo, el manejo de las TIC y la planificación del trabajo no presencial.

\section{REFERENCIAS}

Alfageme González, M.B., El portafolio reflexivo: metodología didáctica en el EEES. Educatio Siglo XXI: 25, 209-226 (2007).

De Miguel Díaz, M., Modalidades de enseñanza centradas en el desarrollo de competencias. Orientaciones para promover el cambio metodológico en el espacio europeo de educación superior. Ediciones de la Universidad de Oviedo, Oviedo (2005).

García-Montalvo, J., Formación y empleo de los graduados de enseñanza superior en España y en Europa. IVIE, Valencia (2001).

García-Valcárcel, A., La tutoría en la enseñanza universitaria y la contribución de las TIC para su mejora. Relieve: 14, 1-14 (2008).

Huber, G.L., Aprendizaje activo y metodologías educativas. Revista de Educación: número extraordinario, 59-81 (2008).

Lázaro, A., La necesidad de la tutoría en la calidad universitaria. Revista Española de Pedagogía: $225,17-42$ (2004).

Martín, P. y Bobb Wolff, L., La implantación de los nuevos grados: propuestas de mejora. Revista de Educación: 356 (en imprenta) (2011). 
Martínez, M. y M. Viader, Reflexiones sobre aprendizaje y docencia en el actual contexto universitario. La promoción de equipos docentes. Revista de Educación: número extraordinario, 213-234 (2008).

Monreal-Gimeno, M.C., Las guías docentes como instrumento de innovación en la enseñanza superior en el marco de la convergencia europea. Educatio: 23, 33-47 (2005).

Moreno, F. y R. Santiago, Formación online. Guía para profesores universitarios. Universidad de La Rioja, Logroño (2003).

Palomares Ruiz, A., El modelo docente universitario y el uso de nuevas metodologías en la enseñanza, aprendizaje y evaluación. Revista de Educación: 355 (en imprenta) (2011).

Ruiz Ruiz, J.M., Evaluación del diseño de una asignatura por competencias, dentro del EEES, en la carrera de Pedagogía: Estudio de un caso real. Revista de Educación: 351, 435-460 (2008).

Villardón, L., Evaluación del aprendizaje para promover el desarrollo de competencias. Educatio: 24, 27-76 (2006). 